\title{
Prenatal diagnosis of LICAM gene mutations in X-linked hydrocephalus
}

\author{
Mami Yamasaki*1, Tomoko shohuda ${ }^{2}$, Hiroaki Sakamoto3, \\ Masahiro Nonaka ${ }^{1}$ and Yonehiro Kanemura ${ }^{2}$
}

\begin{abstract}
Address: ${ }^{1}$ Department of Neurosurgery, Osaka National Hospital, National hospital organization, 2-1-14 Hoenzaka, Chuo-ku, osaka city, Osaka, 540-0006, Japan, ${ }^{2}$ Institute for clinical research, Osaka National Hospital, National hospital organization, 2-1-14 Hoenzaka, Chuo-ku, osaka city, Osaka, 540-0006, Japan and ${ }^{3}$ Department of Pediatric Neurosurgery, Osaka City General Hospital 2-13-22 Miyakojima-hondouri, Miyakojimaku, osaka city, Osaka, 534-0021, Japan

Email: Mami Yamasaki* - yamasaki@onh.go.jp

* Corresponding author
\end{abstract}

from 50th Annual Meeting of the Society for Research into Hydrocephalus and Spina Bifida

Cambridge, UK. 30 August - 2 September 2006

Published: 21 December 2006

Cerebrospinal Fluid Research 2006, 3(SuppI I):S47 doi:I0.II86/I743-8454-3-SI-S47

() 2006 Yamasaki et al; licensee BioMed Central Ltd.

\section{Background}

X-linked hydrocephalus (XLH) (severe type of human L1 syndrome) is now known to be due to mutations in the gene for the neural cell adhesion molecule L1. We performed prenatal diagnosis of L1CAM gene mutations in 5 families. We evaluated effective methods and discussed contribution for the prenatal diagnosis of XLH.

\section{Materials and methods}

We performed a nation-wide L1 gene analysis of patients with hydrocephalus and identified LI gene mutations in 36 families. In these families, five obligate careers were pregnant subsequently and want to perform the L1CAM gene analysis of their fetuses. Genomic DNA was extracted from chorionic villus biopsy (CVB) at from 10 to 15 weeks' gestations. Amplification of the exons and the exon-intron boundaries of the L1 gene was performed by polymerase chain reaction (PCR) Purified PCR amplification products were directly sequenced using the $\mathrm{ABI}$ BigDye $^{\mathrm{TM}}$ Terminator Cycle Sequencing Ready Reaction Kit (Applied Biosystems) and analyzed with a capillary DNA sequencer ABI PRISM ${ }^{\circledast} 310$ Genetic Analyzer.

\section{Results}

1. Two fetuses were male and three were female. L1CAM gene in two males did not have mutations. In three females, one did not carry the mutation in L1CAM gene and two female fetuses had the same L1CAM gene mutation as his mother. 2. Five obligate careers continued their pregnancy and delivered normal babies.

\section{Conclusion}

Prenatal L1gene analyses are useful for the prenatal diagnosis of X linked hydrocephalus. 